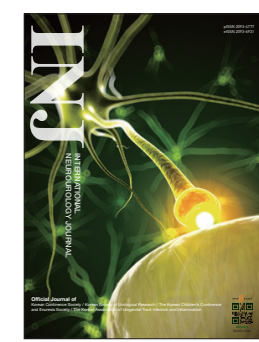

\title{
Recent Patient Health Monitoring Platforms Incorporating Internet of Things-Enabled Smart Devices
}

\author{
Minhee Kang ${ }^{1,2}$, Eunkyoung Park ${ }^{1}$, Baek Hwan Cho ${ }^{1,2}$, Kyu-Sung Lee ${ }^{1,3}$ \\ ${ }^{1}$ Smart Healthcare \& Device Research Center, Samsung Medical Center, Sungkyunkwan University School of Medicine, Seoul, Korea \\ ${ }^{2}$ Department of Medical Device Management and Research, SAIHST, Sungkyunkwan University, Seoul, Korea \\ ${ }^{3}$ Department of Urology, Samsung Medical Center, Sungkyunkwan University School of Medicine, Seoul, Korea
}

Int Neurourol J 2018;22(Suppl 2):S76-82

https://doi.org/10.5213/inj.1836144.072

Due to a mistake in reference by the author submission in this article, an erroneous grant number had been published; this has been corrected as follow:

\section{Corrected Grant Number}

This work was supported by the Korea Health Technology R\&D Project through the Korea Health Industry Development Institute (KHIDI), funded by the Ministry of Health \& Welfare (HI14C3229) and the Bio \& Medical Technology Development Program of the National Research Foundation (NRF) funded by the Ministry of Science (2016M3A9B6919189). 